\title{
Photoacoustic tomography setup using LED illumination
}

Leskinen, Jarkko, Pulkkinen, Aki, Tick, Jenni, Tarvainen, Tanja

Jarkko Leskinen, Aki Pulkkinen, Jenni Tick, Tanja Tarvainen, "Photoacoustic tomography setup using LED illumination," Proc. SPIE 11077, Opto-Acoustic Methods and Applications in Biophotonics IV, 110770Q (19 July 2019); doi: 10.1117/12.2526723

SPIE. Event: European Conferences on Biomedical Optics, 2019, Munich, Germany 


\title{
Photoacoustic tomography setup using LED illumination Jarkko Leskinen,,${ }^{\mathrm{a},}$ Aki Pulkkinen, ${ }^{\mathrm{a}}$ Jenni Tick, ${ }^{\mathrm{a}}$ Tanja Tarvainen ${ }^{\mathrm{a}}$ ${ }^{a}$ University of Eastern Finland, Department of Applied Physics, Yliopistonranta 1F, Kuopio, Finland, 70210
}

\begin{abstract}
Photoacoustic tomography (PAT) is a hybrid imaging modality that combines optical contrast with ultrasound resolution. Most of the PAT configurations are based on high-energy solid-state lasers such as Nd:YAG laser. In this work, a PAT system that uses light-emitting diode (LED) as a light source is introduced. The system is designed so that the imaged target can be stationary. The target is illuminated by a LED light source from one side and the pressure wave is measured using an acoustic transducer that is rotated around the target. Image reconstruction is based on Bayesian approach to illposed inverse problems. The system was tested with light absorbing targets also in limited-view and sparse angle measurement situations. The results show that LED-based instrumentation and advanced reconstruction methods can form a potential PAT system that can also be applied in limited-view and sparse angle photoacoustic tomography.
\end{abstract}

Keywords: photoacoustic tomography, limited-view measurements, light-emitting diode, LED, inverse problems, Bayesian methods.

\section{INTRODUCTION}

Photoacoustic tomography (PAT) is a novel hybrid imaging modality that combines the sub-millimeter spatial resolution of ultrasound (US) imaging and excellent sensitivity and contrast of optical imaging. In PAT, pulsed light is directed into the imaged target that absorbs the energy of the light. The absorbed energy results in macroscopically subtle temperature elevation that results in expansion of the material and a propagating ultrasonic wave. This wave can be measured on the boundary of the target. A photoacoustic image is then reconstructed from the recorded acoustic waveforms using tomographic reconstruction methods ${ }^{1,2}$. Currently, PAT is applied, for example, in imaging cancer and tissue vasculature and in pre-clinical small animal studies ${ }^{2}$.

Most of the PAT systems use large and energetic solid-state lasers, such as Nd:YAG lasers with an optical parametric oscillator (OPO), or dye lasers, to generate illuminations for photoacoustic experiments. Such lasers enable light pulses that have duration of some nanoseconds, energies of tens or even hundreds of millijoules, and widely tunable optical wavelength from visible to infrared. These factors result in deep imaging depths, wide-frequency band acoustic pulses, and direct opportunity for spectroscopic imaging based on a single optical output. However, the devices are also expensive and bulky. The manufacturers have reacted to this by developing systems encompassing the laser, power supply, and cooling into a single unit thereby making the illumination system more robust. However, the systems are still noisy, require large footprint, and are expensive. They may not enable PAT to become an everyday imaging method such as US imaging as the total cost of the PAT system also includes, in addition to the light source, receiver instrumentation to detect US waves.

Alternatives for the laser sources for photoacoustic imaging have been studied. Broadband xenon lamp providing $1 \mu$ s and $3 \mathrm{~mJ}$ pulses have been suggested and tested as an affordable technique for deep tissue imaging ${ }^{3}$. Perhaps the most promising candidates to replace high-energy lasers are laser diodes and light-emitting diodes (LED). Several studies have applied laser diodes as a light source for photoacoustic imaging ${ }^{4-12}$. The diodes can be stacked to form arrays that can produce energies in a millijoule range ${ }^{8,10}$. Pulse durations range from tens to hundreds of nanoseconds. The diodes can be operated with pulse repetition rates in a kilohertz range as opposed to solid-state lasers operating typically in tens of hertz.

*jarkko.leskinen@uef.fi

Opto-Acoustic Methods and Applications in Biophotonics IV, edited by Vasilis Ntziachristos,

Roger Zemp, Proc. of SPIE-OSA Vol. 11077, 110770Q · @ 2019 SPIE-OSA

CCC code: $1605-7422 / 19 / \$ 21 \cdot$ doi: $10.1117 / 12.2526723$

Proc. of SPIE-OSA Vol. 11077 110770Q-1 
The high repetition rate not only enable high frame rate imaging, but it can be applied to increase the signal-to-noise ratio through signal averaging therefore compensating the lower optical energy ${ }^{13}$.

Currently, the most powerful laser diodes operate in the near-infrared wavelengths around 900 to $1000 \mathrm{~nm}$ and not in the visible range, which is the most interesting and widely applied in biomedical photoacoustic imaging of tissue and vascularity ${ }^{14,15}$. Recently, Stylogiannis et al. reported that continuous wave laser diodes can offer suitable wavelengths for photoacoustics ${ }^{15}$. In the study, overdriven laser diodes were used to produce approximately $10 \mathrm{~ns}$ pulses with energies up to $200 \mathrm{~nJ}$ per pulse in the visible and near-infrared range enabling superficial photoacoustic imaging.

Powerful LEDs can be found in wavelengths that are relevant to photoacoustic imaging ${ }^{14,16-21}$. Typically, pulse durations in photoacoustic studies have ranged from tens to hundreds of nanoseconds and pulse repetition rates have varied from hundreds of $\mathrm{Hz}$ to tens of $\mathrm{kHz}$. Energy extracted from a single LED has raised from hundreds of nanojoules (40 ns pulse) ${ }^{16}$ up to $22 \mu \mathrm{J}$ (500 ns pulse) ${ }^{17}$. In the LED arrays, energies up to several hundreds of $\mu \mathrm{J}$ ( $70 \mathrm{~ns}$ pulse) have been reported

${ }^{21}$. The imaging depths are usually limited to less than one centimeter, but submillimeter resolution at the depth of $2-3 \mathrm{~cm}$ have been achieved in phantoms ${ }^{19,21}$. Imaging frame rates as high as $500 \mathrm{~Hz}$ have been reported ${ }^{20}$. Furthermore, LEDs having different operation wavelengths can be combined to form a spectroscopic measurement system ${ }^{14,19}$. Cost of a LEDbased system is low compared to solid-state pulsed lasers and, despite the disadvantages such as lower energy limiting the imaging depth, the LED illuminations may offer PAT a path for a wider everyday use.

In this study, a LED-based photoacoustic tomography system is introduced. Majority of the current LED based photoacoustic systems are imaging setups which apply planar measurement geometry in which the light exposure and sound reception are made on a single plane on the surface of the target providing planar (projection) images ${ }^{19-21}$. An exception to this is the tomographic system introduced by Allen and Beard ${ }^{14}$. In their system, a target is placed between a light source and an ultrasound transducer. The target is rotated, and measurements are performed at multiple detection angles. Then, a tomographic image is reconstructed from these measurements. The study shows that LEDs can be utilised in photoacoustic tomography. However, in practical applications, it may not be possible to rotate the target or perform the measurements from multiple directions. Therefore, in this work, a system for photoacoustic tomography, in which the imaged target and light source are stationary while the sound detecting receiver is rotated at various positions, is developed. Image reconstruction is based on a Bayesian approach ${ }^{22-24}$. This enables tomographic reconstruction also in limited-view and sparse angle measurement geometries.

\section{MATERIALS AND METHODS}

Figure 1 illustrates the photoacoustic tomographic measurement system. The setup was constructed around a plastic water tank placed on a rigid table. An optically clear Mylar window was positioned at one side of the tank to allow LED light to pass into the tank. The tank had external dimensions of $60 \times 40 \times 20 \mathrm{~cm}$ (width $\times$ depth $\times$ height) and it was filled with degassed deionized water (temperature $19-21{ }^{\circ} \mathrm{C}$, corresponding to speed of sound $1479-1485 \mathrm{~m} / \mathrm{s}{ }^{25}$ ) during the experiments. A 3D positioner was used to rotate the US transducer around the imaged target inside the tank. Another 3D positioner was used to hold an imaged sample.

\subsection{LED light source}

A LED (model SST-90-R, Luminus Devices, MA, USA) (Fig. 2(a)-(b)), which operates at $617 \mathrm{~nm}$ wavelength, was driven in a pulsed mode and over-current during the experiments ${ }^{26}$. The LED type was previously used by Allen and Beard ${ }^{14}$. The drive current of $50 \mathrm{~A}$ for the LED was generated using a commercial laser driver module (model LDP-V 50-100 V3, Picolas, Germany). The driver was triggered using an external waveform generator (model 33250A, Agilent, CA, USA) which fed $5 \mathrm{~V}$ pulse (duration $200 \mathrm{~ns}$, pulse repetition frequency $200 \mathrm{~Hz}$ ) for the driver. The external trigger was used to adjust the nominal duration and pulse repetition frequency of the light. The light was collimated and focused using two aspherical lenses (numerical aperture 0.76, diameter $50.8 \mathrm{~mm}$, focal length $32 \mathrm{~mm}$; model ACL50832U, Thorlabs, NJ, USA) attached to lens holders (model $\mathrm{CH} 2 \mathrm{~B}$, Thorlabs). The distance between the last lens and the imaged target was approximately $40 \mathrm{~mm}$ so that the target was placed beyond the optical focal point. This was to ensure that the whole sample was illuminated. The duration of the LED pulse was measured using a silicon PIN photodiode (model S1223, Hamamatsu 
(a)

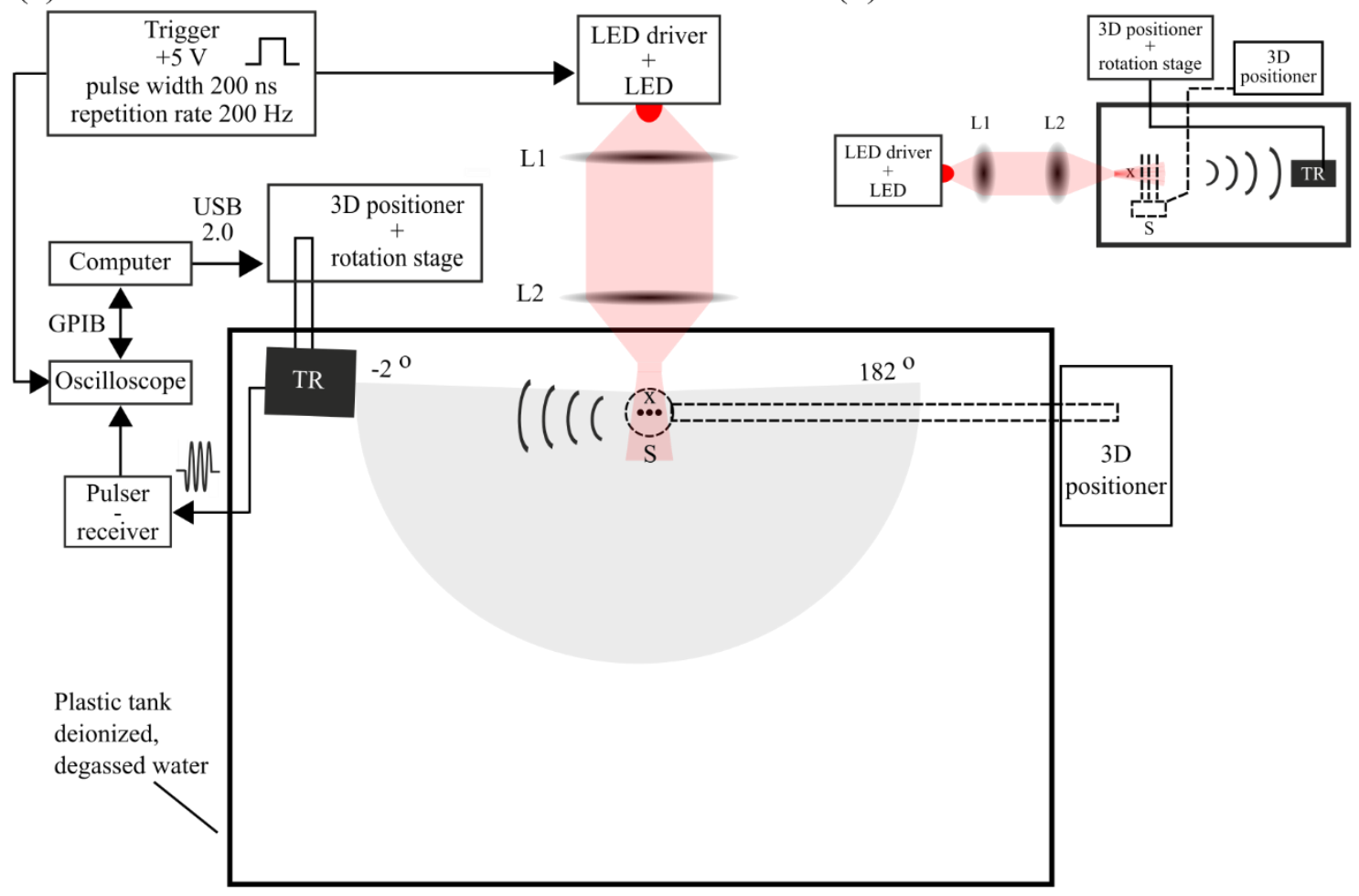

(b)

Figure 1. Schematic illustration of the photoacoustic setup showing (a) the top view and (b) side view. L1, L2 = optical lenses; $\mathrm{S}=$ sample; $\mathrm{TR}=$ ultrasound transducer; $\mathrm{x}=$ center of rotation.

Photonics, Japan) connected to a digital oscilloscope (model 6051A WR, LeCroy, NY, USA) through a biasing circuitry. These measurements were made using the same setup as in the tomographic experiments, except that the tank was not filled with water. The measured photodiode waveform is shown in Fig. 2(c).

The rise time of the light pulse (defined as the time for the signal to rise from $10 \%$ to $90 \%$ of the maximum value) was found to be $51 \mathrm{~ns}$. The pulse fall time (defined as the time for the signal to fall from $90 \%$ to $10 \%$ of the maximum value) was found to be $265 \mathrm{~ns}$. The full width at half maximum (FWHM) time was $280 \mathrm{~ns}$. Time value $t=0$ indicates the trigger time, and approximately $40 \mathrm{~ns}$ delay is due to the delays in transmission and detection circuits. The optical energy of the LED pulse was measured using a pyroelectric sensor (model 919E-200U-8-25K, Newport, CA, USA) connected to an energy meter (model 841-PE-USB, Newport). The energy was measured inside an empty tank at the focal point. Fiveminute average and standard deviation were found to be $5.36 \pm 0.02 \mu \mathrm{J}$. The spatial width of the light beam was estimated using a digital camera (model G12, Canon, Japan) and sheet of laser copy paper $\left(80 \mathrm{~g} / \mathrm{m}^{2}\right)$ as a light screen. The paper screen had printed scaling, and it was placed on the path of the LED beam using the 3D positioner inside the empty tank. A digital image was taken of the screen when the screen was at the same position as the sample during a PAT measurement. The red channel of the image was analyzed in MATLAB (version R2017b, The MathWorks, Inc., MA, USA). The beam was found to have approximately symmetric parabolic shape with FWHM value of $12.7 \mathrm{~mm}$.

\subsection{Ultrasound detector}

The pressure waves were detected using a circular piston transducer (nominal diameter $12.5 \mathrm{~mm}$; model V306, Olympus NDT, MA, USA). The transducer was fixed to a computer-controlled rotation stage (model 8MR190-2-4233-400mA and model 8SMC1-USBh controller, Standa, Vilnius, Lithuania) enabling rotation of the transducer around the target. 
(a)

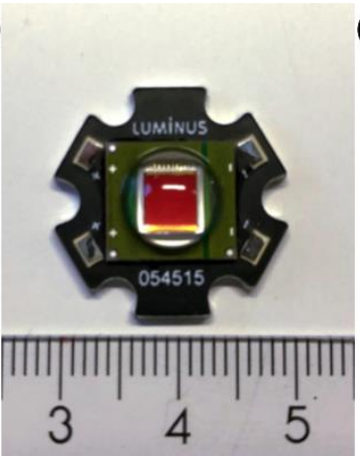

(b)

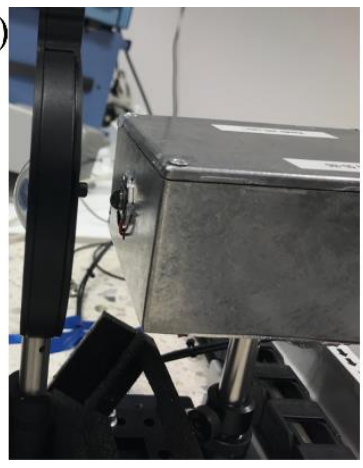

(c)

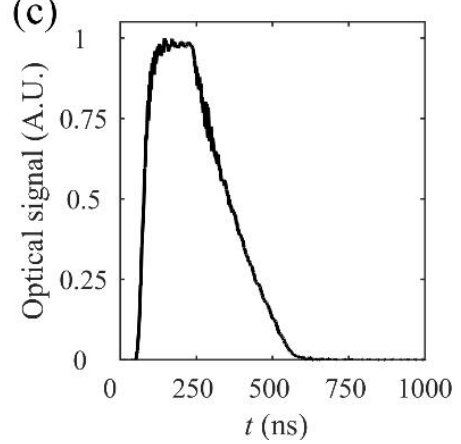

Figure 2. (a) Photograph of the LED used in the setup including the hemispherical lens covering the $3 \mathrm{~mm} \times 3 \mathrm{~mm}$ element. The ruler scaling is in centimeters. (b) The LED driver was housed inside a metallic box to alleviate electromagnetic interference. The metal box also served as a rigid back-plate for the LED with thermal transfer properties. (c) The measured optical waveform of the LED pulse. The waveform was normalized with respect to its maximum value.

The rotation stage was connected to three-axis linear positioner (Time and Precision, Basingstoke, UK). In this study, the angular measurement range was $184^{\circ}$ ranging from $-2^{\circ}$ to $182^{\circ}$ around the sample, and the angular step was $1^{\circ}$. The distance between the transducer and center of rotation was approximately $70 \mathrm{~mm}$ and it was adjusted using the method described in Sec. 2.3.

During the measurements, the transducer signal was amplified ( $40 \mathrm{~dB}$, gain $40 \mathrm{~dB}$ ) and filtered (pass band from $100 \mathrm{kHz}$ to $10 \mathrm{MHz}$ ) using a pulser-receiver (model $5800 \mathrm{PR}$, Olympus NDT). The electric signal was then coupled to a digital oscilloscope (model 6051A WR, LeCroy) and stored for analysis and reconstructions. Each stored waveform was an average of 1024 waveforms, and the sampling rate was $100 \mathrm{MHz}$. The transducer rotation and the waveform detection were synchronized using a custom LabVIEW-program (version 2017, National Instruments, TX, USA) through USB 2.0 and GPIB-buses using the workstation with Windows 7 (Microsoft, WA, USA) operating system.

The transducer's reception beam size was measured using a PVDF needle hydrophone (active area $0.2 \mathrm{~mm}$, Precision Acoustics, Dorchester, UK) connected to a US measurements system (UMS V 1.3.5., Precision Acoustics). The measurements were made at a $70 \mathrm{~mm}$ distance from the transducer inside a water-filled tank and in a free field condition by driving the transducer using the pulser. This distance corresponds to the location beyond the last axial maximum point for the transducer and results in a symmetric acoustic field in radial directions. The acoustic beam width (FWHM) was 6.6 $\mathrm{mm}$ at this distance. The acoustic pressure varied 5\% when the distance from the transducer was varied between 60 and $80 \mathrm{~mm}$ indicating slowly descending pressure profile in the axial direction. The measured central frequency of the transducer was $2.4 \mathrm{MHz}$ and FWHM was from 1.4 to $3.4 \mathrm{MHz}$.

\subsection{Targets for imaging}

The targets were made of plastic microcapillary tubes (PETG; Globe Scientific, NJ, USA) that had length, inner diameter and outer diameter of $75 \mathrm{~mm}, 0.85 \mathrm{~mm}$, and $1.55 \mathrm{~mm}$, respectively (Fig. 3(a)). The tubes were filled with Indian ink (art. nro. 44257000, Royal Talens, Apeldorn, the Netherlands) solution. The ink was diluted in deionized and degassed water using $2 \mathrm{ml}$ Eppendorf vials and had concentrations of $0.1 \%$ and $0.2 \%$ (volume fraction). Prior to the experiments, the vials were sonicated for 30 minutes in an ultrasonic bath to homogenize the dilutes ${ }^{27}$. The concentrations of $0.1 \%$ and $0.2 \%$ correspond to absorption coefficients of approximately $3.4 \mathrm{~cm}^{-1}$ and $7.5 \mathrm{~cm}^{-1}$, respectively ${ }^{27}$. The tubes were filled with the ink solution and sealed using bee-wax. The filled tubes were placed on an aluminum holder (Fig. 3(b)-(c)) that had machined holes for the tubes in one end and M6 inner bore in another end for fixing to a manual 3D positioner (model 7T173-20-50, Standa). With this positioner, the target was placed into the tank. Prior to the experiments, in order to adjust the transducer position, a $1 \mathrm{~mm}$ diameter steel pin attached to the aluminum holder was fixed on the manual positioner. First, this pin was moved to the center of the light beam. Then, the US transducer was operated in a pulse-echo mode, and the reflection from the steel pin was recorded at angles from $-2^{\circ}$ to $182^{\circ}$. By inspecting the time-of-flight and by adjusting the transducer position using the three-axis linear slide positioner, the center of the pin could be placed at the center of the 
(a)

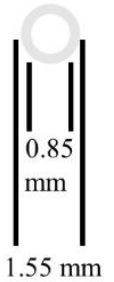

(b)

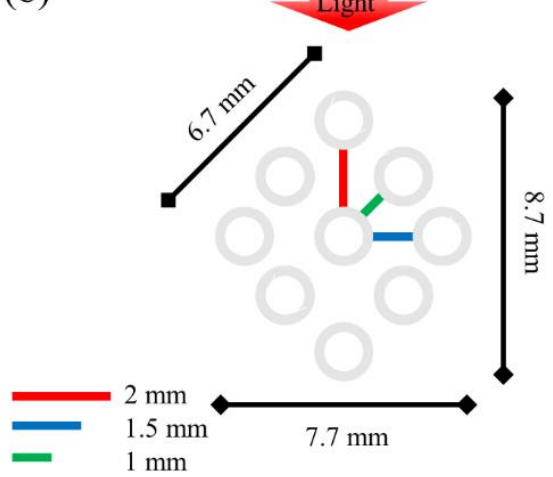

(c)

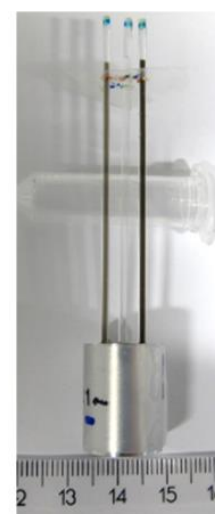

Figure 3. (a) Inner and outer diameter of the capillary tube. (b) The gaps between the machined holes for the tubes in the tube holder. (c) Photograph illustrating the aluminum sample tube holder and tubes inserted into the holder. Ink concentration in the tubes from left to right are $0.1 \%, 0 \%$, and $0.2 \%$, respectively. The ruler scaling is in centimeters.

rotation. The distance between the transducer and center of rotation (center of the target) was $70.31-70.36 \mathrm{~mm}$ over $184^{\circ}$ range. After this, the target was moved $3 \mathrm{~mm}$ inside the arc of rotation (i.e. away from the LED) so that the entire target was inside the transducer's reception field.

\subsection{Image reconstruction}

Photoacoustic images, i.e. the initial pressure caused by the absorption of the light, were reconstructed using a Bayesian framework for inverse problems using MATLAB (version R2015b, The MathWorks, Inc.). Following the approach described in ${ }^{24,28}$, i.e. modelling all parameters as random variables and formulating the forward model with an additive noise model and a prior model for the unknowns, the posterior probability density function describing the solution of the inverse problem can be written as

$$
\pi\left(p_{0} \mid p_{t}\right) \propto \exp \left\{-\frac{1}{2}\left\|L_{e}\left(p_{t}-K p_{0}-\eta_{e}\right)\right\|^{2}-\frac{1}{2}\left\|L_{p_{0}}\left(p_{0}-\eta_{p_{0}}\right)\right\|^{2}\right\}
$$

where $p_{t}$ is a vector of the measured acoustic pressure waves, $p_{0}$ is the initial pressure within the medium, $K$ is the discrete forward model for the pressure propagation which maps the initial pressure distribution to the measurable data, $\eta_{e}$ and $L_{e}$ are the mean of the noise and the Cholesky decomposition of the inverse of the noise covariance matrix, and $\eta_{p_{0}}$ and $L_{p_{0}}$ are the mean of the prior and the Cholesky decomposition of the inverse of the prior covariance matrix. In this work, the forward model was the linear wave equation

$$
\left(\frac{\partial^{2}}{\partial t^{2}}-c^{2} \nabla^{2}\right) p(r, t)=0
$$

with initial conditions

$$
\begin{gathered}
p(r, t=0)=p_{0}(r) \\
\frac{\partial}{\partial t} p(r, t=0)=0
\end{gathered}
$$

where $r$ indicates spatial position, $c$ is the speed of sound, and $t$ indicates time. The wave equation was solved using a $k$ space time-domain method with k-Wave MATLAB toolbox ${ }^{29}$. The forward model $K$ included the simulation of bandpass filter behavior of the transducer with $-6 \mathrm{~dB}$ filter cutoff frequencies 1.4 and $3.4 \mathrm{MHz}$. For the prior model, OrnsteinUhlenbeck process ${ }^{30}$ was used with a mean $\eta_{p_{0}}=0.015$, standard deviation $\sigma_{p_{0}}=0.005$ and characteristic length scale $\ell=0.85 \mathrm{~mm}$. The mean of the posterior, which in this case corresponds to the maximum a posteriori estimate, was solved. It was computed using a matrix free approach and utilizing the adjoint of the wave equation as described in ${ }^{28,31}$. 
For the numerical implementation of the 2D reconstruction of the initial pressure, a computation domain of size $14.6 \times 8.2$ $\mathrm{cm}$ was considered. It was discretized into $730 \times 410$ pixels, i.e. pixel width $0.2 \mathrm{~mm}$. A perfectly matched layer (PML) with thickness of 3.8 and $2.2 \mathrm{~mm}$ (19 and 11 pixels) was positioned outside the computation domain to avoid computational artifacts due to boundary reflections. The measured signal was downsampled from $100 \mathrm{MHz}$ to $20 \mathrm{MHz}$ for the reconstructions. The noise statistics were determined from the measurements by determining the mean and standard deviation at each detector position from a 181 time point timeframe of the measured signal before the triggering of the light pulse. The signal after the trigger was taken into reconstructions and contained 1790 temporal samples. The distance between the transducer and center of rotation was set to $70.3 \mathrm{~mm}$ in the computations.

In addition to visual inspection of the reconstructions, diameters of the tubes were analyzed by forming profiles across the tube centers on the reconstructed images and calculating the tube diameters as a FWHM values around the peak. The final value for the tube diameter was calculated as an average of the tubes in the reconstructed image. The same procedure was used to estimate the distance between the tube centers, except that the distance was calculated between the peak values of the profiles.

\section{RESULTS}

\subsection{Photoacoustic signal}

First, a target having three micro capillary tubes containing $0.1 \%, 0.2 \%$, and $0 \%$ (pure water) ink concentration (Fig. 4(a)) was investigated. Amplitude of the received acoustic waveform was less than one $\mathrm{mV}$, underlining the small level of signals present at the measurements (Fig. 4(b)). Based on the frequency spectrum (Fig. 4(c)), the measured signal contained frequencies that were mostly less than $1.5 \mathrm{MHz}$, which is not the optimal detection bandwidth of the transducer. The tube containing water was not visible in the sinogram (Fig. 4(d)) nor in the reconstructed image (Fig. 4(e)) indicating that tube walls were not active sound sources, and thus contrast was obtained through ink. Some reverberation was evident in the sinogram indicating acoustic reflection between the tubes. In the reconstructions, measured data from 185 measurement angles from $-2^{\circ}$ to $182^{\circ}$ around the sample were used (i.e. $1^{\circ}$ step). The reconstructions were computed using the sound speed of $1482.7 \mathrm{~m} / \mathrm{s}$. The tubes containing different ink concentrations are clearly identifiable in the reconstructed images (Fig. 4(e)).

\subsection{Multiple absorbers and different alignment of targets}

Three tubes filled with $0.1 \%$ ink solution were investigated. The transducer was rotated from $-2^{\circ}$ to $182^{\circ}$ using $1^{\circ}$ step size. Three different rotation angles of the tubes with respect to the illumination direction were inspected (Fig. 5(a) - (c)). The nominal distance between the tube centers was $2.5 \mathrm{~mm}$, and the tubes were separated by $1 \mathrm{~mm}$. In the reconstructions, speed of sound of $1482.4,1482.1$ and $1479.6 \mathrm{~m} / \mathrm{s}$ were used for the tubes in perpendicular, approximately $45^{\circ}$ rotation and in parallel with the light beam, respectively, because of the different water temperatures in the measurements. After analyzing the reconstructed images (Fig. 5), the estimated tube diameters were $1 \pm 0.1 \mathrm{~mm}, 1 \pm 0.2 \mathrm{~mm}$, and $1 \pm 0.1 \mathrm{~mm}$ for the tubes in perpendicular, with $45^{\circ}$ rotation, and in parallel with the light beam, respectively. For the parallel orientation (Fig. 5(c)), the diameter is an average of two uppermost tubes because of the low signal level at the lowest tube. The calculated center-to-center distances for the tubes were $2.4 \pm 0.3 \mathrm{~mm}$ (perpendicular), $2.3 \pm 0.1 \mathrm{~mm}\left(45^{\circ}\right.$ rotation), and $2.4 \pm 0.1 \mathrm{~mm}$ (parallel).

Following the three tube experiments, a target consisting of nine tubes filled with $0.1 \%$ ink solution was investigated. In the reconstruction, the speed of sound in the medium was set to $1480.3 \mathrm{~m} / \mathrm{s}$. The reconstruction is shown in Fig. 6 . In the reconstruction, only the tubes closest to the illumination side are clearly identifiable. The tubes off the central line or farther from the source were difficult to separate which is due to a low absorbed optical energy density within them, i.e. most of the light has been absorbed by the tubes closest to the light source. 
(a)

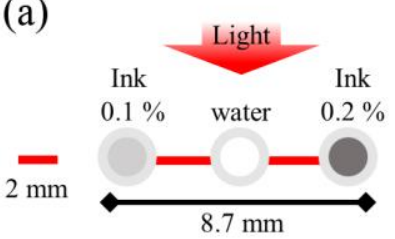

(b)
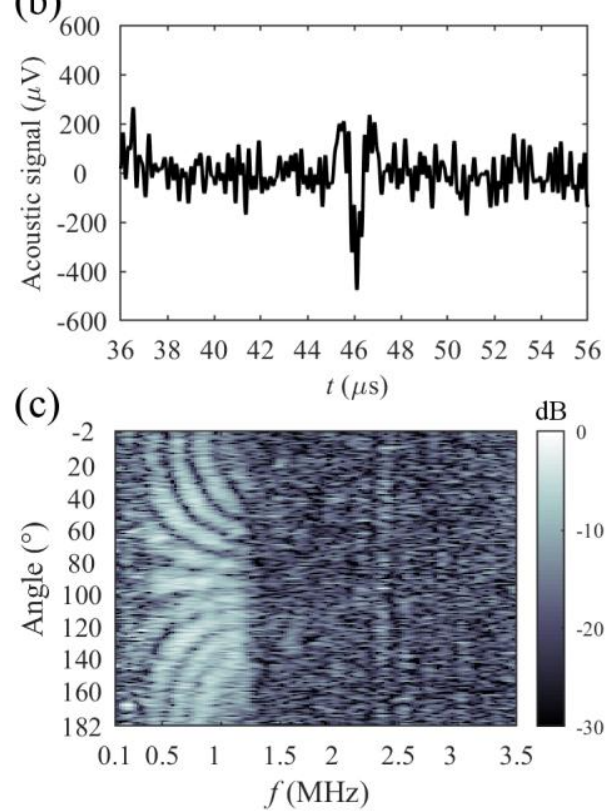

(d)

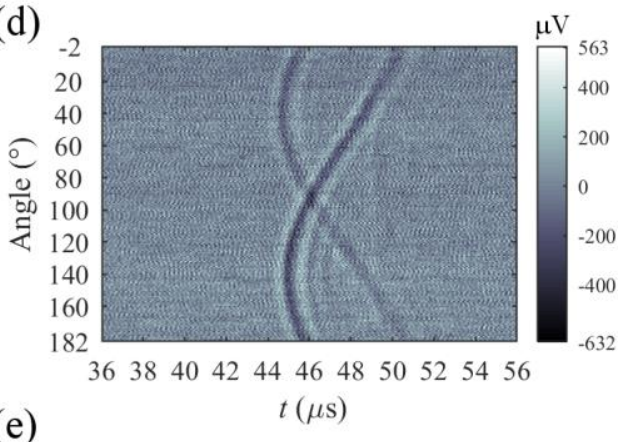

(e)

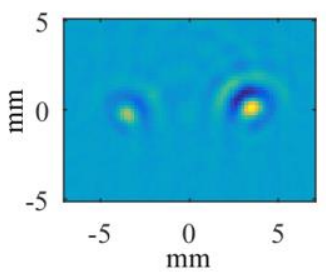

Figure 4. (a) An illustration of sample tubes containing ink concentrations $(0.1 \%$ and $0.2 \%)$ and pure water. (b) The raw ultrasound waveform captured at an angle of $90^{\circ}$. (c) The frequency spectrum as a function of the measurement angle. The decibel $(\mathrm{dB})$ value are calculated with respect to the maximum value in the spectrum. (d) The sinogram of the captured waveforms. (e) A reconstructed image of the capillary tubes obtained using the Bayesian approach.

\subsection{Reconstructions using sparse angle data}

In order to study the performance of the system in a sparse angle imaging situation, photoacoustic images were reconstructed using only part of the data measured from the three ink filled tubes with a position with $45^{\circ}$ rotation against the illumination light. The reconstructions were computed using 185, 93, 37, 19, 10 and 7 measurement angles distributed uniformly between $-2^{\circ}$ and $182^{\circ}$ around the target (i.e. angles with the separation of $1^{\circ}, 2^{\circ}, 5^{\circ}, 10^{\circ}, 20^{\circ}$ and $30^{\circ}$ ) are shown in Fig. 7 (a) - (f). As it can be seen, the methodology is capable to visualize the locations of the tubes even if the number of measurements is reduced significantly. In fact, in this case, the image quality seems qualitatively equally good with 185 , 93 and 37 measurement angles, and even with 19 angles the tubes can be visually distinguished from the artefacts. This indicates that using advanced methods for image reconstruction can enable sparse and limited angle imaging without compromising the accuracy of the reconstructions. 
(a)
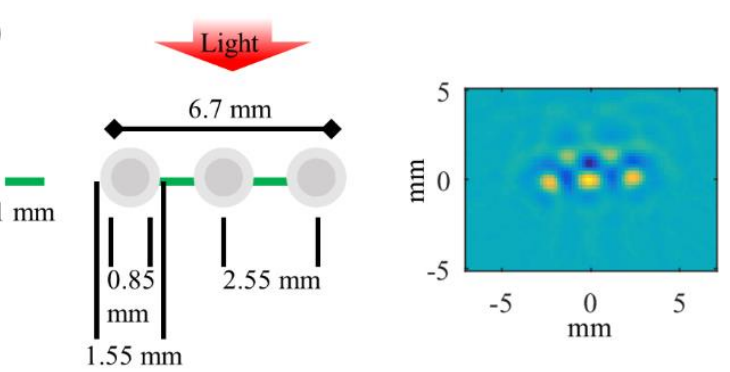

(b)

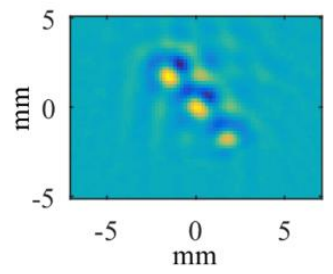

(c)

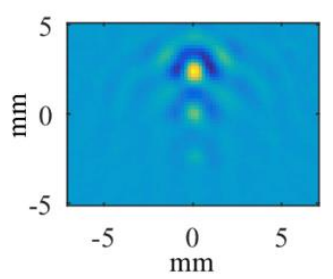

Figure 5. Reconstructed photoacoustic images of three ink (0.1\%) filled tubes when the direction of the tube pattern was in (a) perpendicular, (b) at approximately $45^{\circ}$ angle and (c) in parallel relative to the light source.

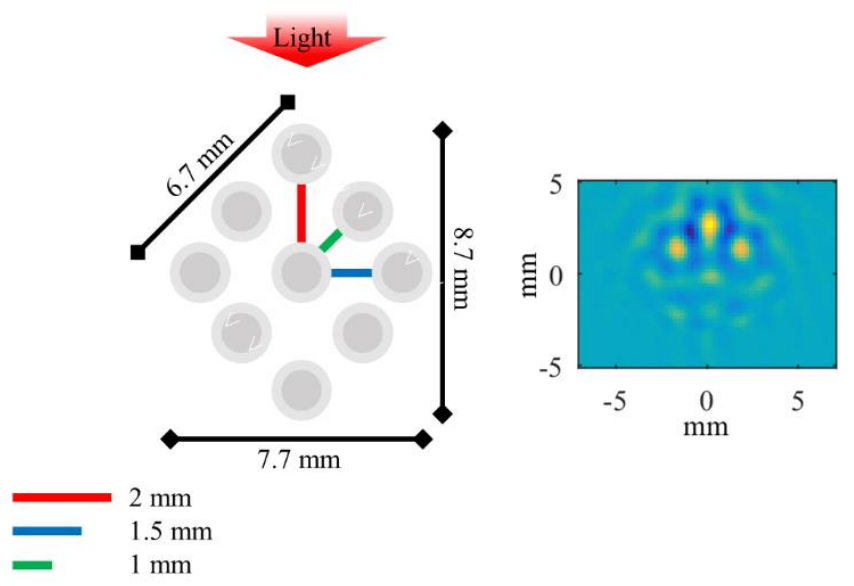

Figure 6. Reconstructed photoacoustic images of nine ink $(0.1 \%)$ filled tubes positioned in the form of a diamond shape.

Proc. of SPIE-OSA Vol. 11077 110770Q-8 

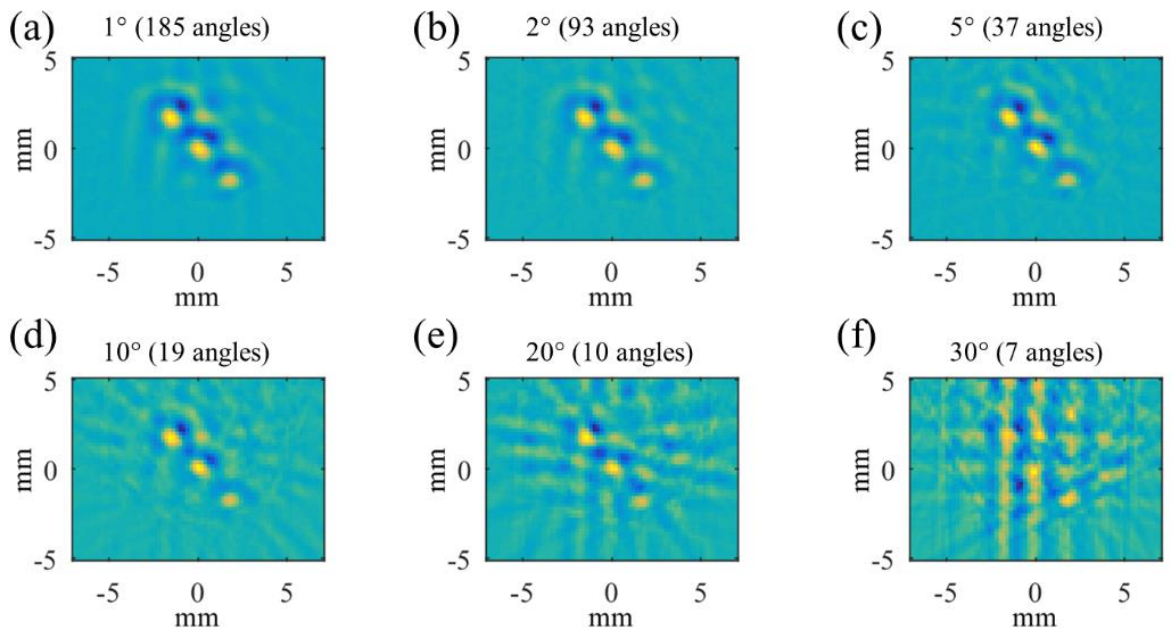

Figure 7. (a) - (f) Reconstructions from sparse angle measurements. The separation between the measurement angles and the number of the measurement angles are shown on the top of each reconstruction.

\section{DISCUSSION AND CONCLUSIONS}

In the developed PAT system, the light beam pattern at the optical focus had rectangular cross-section and beam width of approximately $4.5 \mathrm{~mm}$. Therefore, the maximum photon fluence for the current system is approximately $26 \mu \mathrm{J} / \mathrm{cm}^{2}$. In our case, as the samples were larger than the focal spot size, the samples were positioned behind the focal point to ensure that the whole target was illuminated. The intensity pattern of the light beam beyond the focal point was parabolic (FWHM area and approximative fluence $1.27 \mathrm{~cm}^{2}$ and $4.3 \mu \mathrm{J} / \mathrm{cm}^{2}$, respectively) which at least partly explains the non-homogenous amplitudes that are visible in the reconstructed images (see Fig. 5(c), parallel orientation). Specifically, the tubes placed at the center of the sample holder received the highest fluence resulting in the highest acoustic pressures. In the setup, the use of collimating and focusing lenses enabled larger separation between the tank wall and sample which resulted in elimination of artefacts due to the sound reflections between the target and the tank wall. More uniform lighting with the expense of fluence would be possible by collimating light with a diffuser. The stability of the LED pulse energy was found to be good as the energy fluctuation was less than $0.5 \%$. Hariri et al. ${ }^{19}$ have reported power fluctuations of approximately $0.1 \%$ or less up to $4 \mathrm{kHz}$ pulse repetition rate for their multielement LED source. These observations underline the good energy stability of LED sources.

With LEDs, spectral shift can occur depending on the LED type and drive conditions. According to the manufacturers data sheet ${ }^{26}$, the LED used in the setup has dominant wavelength variation from 615 to $619 \mathrm{~nm}$. This can be regarded as a variation among the individual LED chips. In addition to this, the wavelength shift may be induced through heating or over-current ${ }^{32,33}$. With the LED of this study, the manufacturer specified shifts due to the current and temperature as +1 $\mathrm{nm}(3.15 \mathrm{~A}$ to $9 \mathrm{~A})$ and $+3.5 \mathrm{~nm}\left(25^{\circ} \mathrm{C}\right.$ to $\left.80^{\circ} \mathrm{C}\right)$, respectively. Elevated LED temperatures are accompanied with a drop in optical energy ${ }^{26,32}$. It is likely that the spectral shift during the measurement at 50 A current was low due to the low duty cycle $(\approx 0.006 \%)$. However, it should be noted that in a study of Willert et al. ${ }^{32}$, a pulsed green LED was found to have close to $14 \mathrm{~nm}$ shift (approximately from 524 to $510 \mathrm{~nm}$ ) with some spectral widening when drive current was elevated from $4 \mathrm{~A}$ to $55 \mathrm{~A}$. Respective pulse width was varied from $10 \mu \mathrm{s}$ to $1 \mu \mathrm{s}$ at a constant $1 \mathrm{kHz}$ pulse repetition rate. These shifts may need to be considered in accurate spectroscopic measurements, but they may not be wide enough so that a single LED could be applied as a wavelength-tunable light source. Fortunately, advanced new LED technologies may offer solution for wavelength tuning in future ${ }^{34}$, so that single LEDs could be applied as a wide spectrum light source.

The 185-angle measurements with 1024 waveform averaging took approximately 30 minutes. This could be speeded up by using higher pulse repetition rate. A focused transducer ${ }^{14,35}$ could be used to improve the signal-to-noise ratio or reduce the waveform averaging. Focusing allows the use of transducers which have large active area with frequencies of several 
$\mathrm{MHz}$, but still reasonable measurement distances compared to the piston sources with long far-field distance. A short transducer-target distance would help to maintain the mechanical structure simple and limits the imaging volume enabling more efficient computational reconstruction. As the transducer is connected to a computerized 3D positioner, volumetric imaging by moving the transducer would also be possible. Customized drive circuits ${ }^{32,36}$ might enable higher drive currents even using this same LED type, resulting in higher optical energies and higher acoustic signals.

Considering improving the contrast of the technique, which was shown to decrease in the presence of multiple absorbers (Fig. 6), multiple LED sources could be positioned sparsely around the target. Efficient focusing of LED sources is difficult in practice so that large number of sources may be difficult to position around the objects due to limited space. However, even some additional light sources at different sides of the target would ensure more uniform illumination of complex targets.

In this work, the image reconstruction was based on the Bayesian approach to ill-posed inverse problems. The approach is not limited to specific geometries such as spherical or cylindrical detection surfaces, and thus it is flexible. Furthermore, imaging in a sparse or limited view setting is possible without compromising the quality of the reconstructions. In the experiments of the paper, the tubes were visible even when the number of measurement angles used in the reconstructions was 37 (Fig. 7(c)). Using a low number of measurement angles decreases the measurement times significantly which increases the suitability of the approach for practical applications. Applications for the affordable low-energy LED systems could include tomographic imaging of skin, superficial tissue, and vasculature, for instance, in the case of a skin cancer screening and visualization.

\section{ACKNOWLEDGEMENTS}

This work has been supported by the Academy of Finland (projects 286247, 314411 and 312342 Centre of Excellence in Inverse Modelling and Imaging), Alfred Kordelin Foundation and Jane and Aatos Erkko Foundation.

\section{REFERENCES}

[1] Beard, P., "Biomedical photoacoustic imaging," Interface Focus. 1(4), 602-631 (2011).

[2] Wang, L. V. and Yao, J., "A practical guide to photoacoustic tomography in the life sciences," Nat. Methods 13(8), 627-638 (2016).

[3] Wong, T. T., Zhou, Y., Garcia-Uribe, A., Li, L., Maslov, K., Lin, L. and Wang, L. V., "Use of a single xenon flash lamp for photoacoustic computed tomography of multiple-centimeter-thick biological tissue ex vivo and a whole mouse body in vivo," J. Biomed. Opt. 22(4), 41003 (2017).

[4] Allen, T. J. and Beard, P. C., "Pulsed near-infrared laser diode excitation system for biomedical photoacoustic imaging," Opt. Lett. 31(23), 3462-3464 (2006).

[5] Kolkman, R. G., Steenbergen, W. and van Leeuwen, T. G., "In vivo photoacoustic imaging of blood vessels with a pulsed laser diode," Lasers Med. Sci. 21(3), 134-139 (2006).

[6] Allen, T. J. and Beard, P. C., "Dual wavelength laser diode excitation source for 2D photoacoustic imaging,"Proc. SPIE 6437, 64371U-1-64371U-9 (2007).

[7] Zeng, L., Liu, G., Yang, D. and Ji, X., "3D-visual laser-diode-based photoacoustic imaging," Opt. Express 20(2), 1237-1246 (2012).

[8] Daoudi, K., van den Berg, P. J., Rabot, O., Kohl, A., Tisserand, S., Brands, P. and Steenbergen, W., "Handheld probe integrating laser diode and ultrasound transducer array for ultrasound/photoacoustic dual modality imaging," Opt. Express 22(21), 26365-26374 (2014).

[9] Wang, T., Nandy, S., Salehi, H. S., Kumavor, P. D. and Zhu, Q., "A low-cost photoacoustic microscopy system with a laser diode excitation," Biomed. Opt. Express 5(9), 3053-3058 (2014). 
[10] Upputuri, P. K. and Pramanik, M., "Performance characterization of low-cost, high-speed, portable pulsed laser diode photoacoustic tomography (PLD-PAT) system," Biomed. Opt. Express 6(10), 4118-4129 (2015).

[11]Erfanzadeh, M., Salehi, H. S., Kumavor, P. and Zhu, Q., "Improvement and evaluation of a low-cost laser diode photoacoustic microscopy system for ovarian tissue imaging,"Proc. SPIE 9708, 97083I-1-97083I-7 (2016).

[12] Hariri, A., Fatima, A., Mohammadian, N., Mahmoodkalayeh, S., Ansari, M. A., Bely, N. and Avanaki, M. R. N., "Development of low-cost photoacoustic imaging systems using very low-energy pulsed laser diodes," J. Biomed. Opt. 22(7), 75001 (2017).

[13] Allen, T. J., Cox, B. T. and Beard, P. C., "Generating photoacoustic signals using high-peak power pulsed laser diodes," Proc. SPIE 5696, 233-242 (2005).

[14] Allen, T. J. and Beard, P. C., "High power visible light emitting diodes as pulsed excitation sources for biomedical photoacoustics," Biomed. Opt. Express 7(4), 1260-1270 (2016).

[15] Stylogiannis, A., Prade, L., Buehler, A., Aguirre, J., Sergiadis, G. and Ntziachristos, V., "Continuous wave laser diodes enable fast optoacoustic imaging," Photoacoustics 9, 31-38 (2018).

[16] Skov Hansen, R., "Using high-power light emitting diodes for photoacoustic imaging,"Proc. SPIE 7968, 79680A179680A-6 (2011).

[17] Allen, T. J. and Beard, P., "Light emitting diodes as an excitation source for biomedical photoacoustics,"Proc. SPIE Photons Plus Ultrasound: Imaging and Sensing 8581, 85811F-1-85811F-9 (2013).

[18] Dai, X., Yang, H. and Jiang, H., "In vivo photoacoustic imaging of vasculature with a low-cost miniature light emitting diode excitation," Opt. Lett. 42(7), 1456-1459 (2017).

[19] Hariri, A., Lemaster, J., Wang, J., Jeevarathinam, A. S., Chao, D. L. and Jokerst, J. V., "The characterization of an economic and portable LED-based photoacoustic imaging system to facilitate molecular imaging," Photoacoustics 9, 10-20 (2018).

[20]Zhu, Y., Xu, G., Yuan, J., Jo, J., Gandikota, G., Demirci, H., Agano, T., Sato, N., Shigeta, Y. and Wang, X., "Light emitting diodes based photoacoustic imaging and potential clinical applications," Sci. Rep. 8(1), 9885-018-28131-4 (2018).

[21] Xia, W., Kuniyil Ajith Singh, M., Maneas, E., Sato, N., Shigeta, Y., Agano, T., Ourselin, S., J West, S. and E Desjardins, A., "Handheld real-time LED-based photoacoustic and ultrasound imaging system for accurate visualization of clinical metal needles and superficial vasculature to guide minimally invasive procedures," Sensors 18(5), E1394 (2018).

[22] Kaipio, J. P. and Somersalo, E., [Statistical and Computational Inverse Problems], Springer, New York (2005).

[23] Tarvainen, T., Pulkkinen, A., Cox, B. T., Kaipio, J. P. and Arridge, S. R., "Bayesian image reconstruction in quantitative photoacoustic tomography," IEEE Trans.Med.Imaging 32(12), 2287-2298 (2013).

[24] Tick, J., Pulkkinen, A. and Tarvainen, T., "Image reconstruction with uncertainty quantification in photoacoustic tomography," J. Acoust. Soc. Am. 139(4), 1951-1961 (2016).

[25] Marczak, W., "Water as a standard in the measurements of speed of sound in liquids," J. Acoust. Soc. Am. 102(5), 2776-2779 (1997).

[26]Luminus Devices Inc., "SST-90-R Product Datasheet," PDS-002760 Rev 01 (2015).

[27] Di Ninni, P., Martelli, F. and Zaccanti, G., "The use of India ink in tissue-simulating phantoms," Opt. Express 18(26), 26854-26865 (2010).

[28] Tick, J., Pulkkinen, A., Lucka, F., Ellwood, R., Cox, B. T., Kaipio, J. P., Arridge, S. R. and Tarvainen, T., "Three dimensional photoacoustic tomography in Bayesian framework," J. Acoust. Soc. Am. 144(4), 2061-2071 (2018).

[29] Treeby, B. E. and Cox, B. T., "k-Wave: MATLAB toolbox for the simulation and reconstruction of photoacoustic wave fields," J. Biomed. Opt. 15(2), 021314 (2010).

[30] Rasmussen, C. E. and Williams, C. K. I., [Gaussian Processes for Machine Learning (Adaptive Computation and Machine Learning)], The MIT Press, Cambridge, MA, USA (2006).

[31] Arridge, S. R., Betcke, M. M., Cox, B. T., Lucka, F. and Treeby, B. E., "On the adjoint operator in photoacoustic tomography," Inverse Probl. 32(11), 115012 (2016).

[32] Willert, C., Stasicki, S., Klinner, J. and Moessner, S., "Pulsed operation of high-power light emitting diodes for imaging flow velocimetry," Meas. Sci. Technol 21(7), 075402 (2010).

[33] Vaskuri, A., Kärhä, P., Baumgartner, H., Kantamaa, O., Pulli, T., Poikonen, T. and Ikonen, E., "Relationships between junction temperature, electroluminescence spectrum and ageing of light-emitting diodes," Metrologia 55(2), S86-S95 (2018). 
[34] Wang, X., Tian, H., Mohammad, M. A., Li, C., Wu, C., Yang, Y. and Ren, T. L., "A spectrally tunable all-graphenebased flexible field-effect light-emitting device," Nat. Commun. 6, 7767 (2015).

[35] Wang, X., Pang, Y., Ku, G., Xie, X., Stoica, G. and Wang, L. V., "Noninvasive laser-induced photoacoustic tomography for structural and functional in vivo imaging of the brain," Nat. Biotechnol. 21(7), 803-806 (2003).

[36] Pulkkinen, A., Leskinen, J. J. and Tiihonen, A., "Ultrasound field characterization using synthetic schlieren tomography," J. Acoust. Soc. Am. 141(6), 4600-4609 (2017). 